\title{
Walking With Another: Rural nurses' experiences of mentoring
}

\section{ABSTRACT}

The aim of this constructivist grounded theory study was to explore rural nurses' experiences of mentoring. Mentoring is often proposed as a solution to the problem of workforce shortages. When and how this occurs in the context of rural nursing has not previously been researched.

Cultivating and growing new or novice rural nurses using supportive relationships such as mentoring was found to be an existing, integral part of experienced rural nurses' practice - initiated by living and working in the same community. A two-part process was identified - getting to know a stranger and walking with another. This article examines the second part of this process, walking with another.

The global problem of workforce for nurses can be defined using the parameters of recruitment and retention rates, 'problems' with which result in staff shortages, particularly of experienced nurse. Creating supportive environments that include developing relationships such as mentoring is a potential solution to local staffing needs that does not require intensive resources. Experienced nurses engaged in clinical practice have the potential to cultivate and grow new or novice nurses - many already do so. Recognising their role and providing support as well as development opportunities will bring about a cycle of mentoring within the workplace.

\section{KEYWORDS}

Grounded theory; Mentors; Nursing; Nursing Workforce; Support; Rural nursing 


\section{INTRODUCTION}

This study aimed to examine rural nurses' experiences of mentoring. The problem of workforce for Australian rural nurses provides the background landscape for this study. Factors impacting on this particular group of nurses are an increased shortage and rapid turnover of skilled nurses, an aging demographic, a poor image of rural nursing and characteristics of rurality such as reduced opportunities for education, professional isolation and distance (Blue 2002, Mills et al. 2005a).

Rural nurses, advocates and academics proposed mentoring as a potential strategy for the problem of workforce (Lindsay et al. 2002). The problem of workforce is delimited by recruitment and retention rates of staff that has led to a shortage of nurses (Dakin et al., 2004). The implementation of mentor development and training for Australian rural nurses followed this problematisation of workforce, funded by the Australian Government (Mills et al. 2005b).

Research into the actual experience of nurse mentoring is scarce, although there is a plethora of publications that deal with the process of mentoring and expected outcomes (Glass \& Walter 2000). Research into the experiences of rural nurse mentors is also limited, with only two small studies identified that discuss the implementation of structured mentoring programs (Hanson \& Hilde 1989, Waters et al. 2003). This article aims to present an aspect of the findings from this study that conceptualises the phenomenon of mentoring as walking with another. These findings will then be discussed in relation to the literature about nurse mentoring and the significance of language in developing supportive relationships. 


\section{BACKGROUND}

A grounded theory about how experienced rural nurses cultivate and grow new or novice rural nurses was developed as an outcome from this study. During the concurrent data generation and analysis phase of this constructivist study there was a focus on the importance of using vivo codes, or codes that serve as 'symbolic markers of participants' speech and meanings' (Charmaz, 2006, p.55). As an outcome of this, the final grounded theory relies on in vivo codes to name both the overall theory and its categories.

The grounded theory of cultivating and growing new or novice rural nurses has three aspects. Initially the context of rural nursing (clinicians usually live and work in the same community) motivates experienced rural nurses to get to know a stranger and walk with another. They do this in order to pass on strategies to manage the implications of what they term live my work to new or novice rural nurses. The implications of living and working in the same community are well recognised in the literature about rural nurses generally (Bushy \& Leipert 2005, Hegney et al. 2002).

Walking with another has three subcategories. Together the mentor and mentee keep things in perspective for the new or novice rural nurses. They do this by creating a safe environment for each other and use a form of language we call nurse chat, to share their histories and experiences. Overall cultivating and growing and in particular walking with another is an ongoing process that does not have a finite end. Rather it is integral to the practice of experienced rural nurses who act as significant others in the reference group of clinical practice. This paper will support with findings the conceptualisation of walking with another, as well as drawing upon contemporary nursing literature to explain it more fully. 


\section{THE STUDY}

Our study design used constructivist grounded theory and relied upon both traditional (Glaser \& Strauss 1967, Strauss \& Corbin 1990, 1998) and more evolved methods (Charmaz 2006, Clarke 2005) to generate data, concurrently analyse these and situate our findings in the broader context of the social world of Australian rural nurses. Reflexivity was a constant thread throughout, and we used reflective memoing to document our experiences (Koch 2006, Mills et al. 2006).

\section{Ethical Considerations}

The research study that this journal article is a part of was approved by the Monash University, Australia, Standing Committee on Ethics in Research Involving Humans (2004/630). It conforms to the provisions of the Declaration of Helsinki in 1995 (as revised in Edinburgh 2000). Written informed consent was obtained from all of the participants who were free to withdraw from the study at any time. Pseudonyms have been used when reporting this study.

\section{Data Analysis}

Constant comparative analysis was used in this study (Strauss \& Corbin 1990). Concurrent data generation and analysis occurred (Glaser \& Strauss 1967). Initially line-by-line analysis of the first two interview transcripts was undertaken. This included the use of theoretical comparisons to raise theoretical sensitivity (Strauss \& Corbin 1998). Codes and categories were identified and compared with each other. Eventually, a storyline was constructed in order to model the grounded theory of cultivating and growing (Corbin \& Strauss 1990). Situational maps and social worlds mapping techniques (Clarke 2005) were used throughout the data generation and 
analysis phase of the study. Situational maps were also used in a second interview as a way of theoretically sampling for data. Computer assisted analysis occurred using Textual Analysis Markup ${ }^{\circledR}$ software by Matthew Weinstein for Mac OSX®, Inspiration 7.5® by Inspiration Inc. and Filemaker Pro $®$ by Filemaker. The literature concerning the problem of workforce for Australian rural nurses was also used as a secondary source of data. We used the analytical heuristic of collective action framing (Benford \& Snow 2000) to trace the influences that the different collective groups of actors in the social world of Australian rural nursing had on participants' constructions of mentoring (Mills et al., 2006).

\section{FINDINGS}

Findings from this study were constructed into a grounded theory about rural nurses' experiences of mentoring. Cultivating and growing describes the core category - which occurs under three conditions: planned face-to-face, accidental faceto-face, and planned distant. Properties of cultivating and growing rural nurses are the names that experienced rural nurses use to describe their relationships with new or novice rural nurses. These range from preceptoring, to accidental mentoring, to mentoring and finally deep friendship. Each property is dimensionalised by time as well as levels of trust and engagement. Participants commonly used the name mentoring when describing cultivating and growing in this study.

Sharing similar values is very influential in developing high levels of trust and engagement between the mentor and mentee. If the mentor and mentee identify similar values and cultural backgrounds in each other it is much easier for them to create a stronger bond. As a part of this, sharing an interest in the common practice area of rural nursing is really important. 
In the first instance supportive relationships such as preceptoring, accidental mentoring and mentoring are prompted by new or novice rural nurses either experiencing a critical incident, or showing potential. Experienced rural nurses initiate one of a range of relationships and begin the process of cultivating and growing by getting to know a stranger. How this relationship develops depends on the bond that they feel with the new or novice rural nurse. Preceptoring and accidental mentoring are short-term relationships that provide guidance and support directed at specific clinical skills or incidents. If the experienced rural nurse and the new or novice nurse don't identify similar values and interests which lead to a bond being developed between the two the relationship can often terminate at this point.

Walking with another is the second part of the process that experienced rural nurses use in cultivating and growing rural nurses. Mentors and mentees who identify strongly with one another often stay close once the purpose of this relationship (supporting and developing the new or novice rural nurse) is complete, transitioning into a deep friendship.

Mary uses her own experiences to explain this, 'When I was doing palliative care I always used the word compassion... because of its Roman roots... it actually means...walking with somebody, and that's how I feel about mentoring. You are actually walking the road with them' (Mary: TM6281).

There are three elements in walking with another, the first of which is the mentor's role in keeping in perspective the mentee's experiences. The second is the use of a common language termed nurse chat to speak of their experiences. The third is rural nurses' integration of mentoring into their practice as an outcome not an end, which describes how cultivating and growing rural nurses is embedded into their practice as an ongoing role rather than a finite experience with one mentee. 


\section{Keeping Things In Perspective}

Rural nurse mentors work with mentees to keep things in perspective through creating a safe environment, role modelling, and being a critical friend. Wyn speaks of mentoring as being '....a release valve, but it's many things. It's also somebody to look at the situation and say "now you know", they put it into perspective for you. That's very important I think' (Wyn: TM 27997).

Fundamental to being able to keep things in perspective under each condition (planned face-to-face, accidental face-to-face and planned distant) is the creation of a safe environment where the mentee can discuss their issues.

\footnotetext{
They can come into a safe environment... [where] they can express how they're feeling... [their] doubts about nursing... [they need to] know that you will keep it safe...because tomorrow "I might change my mind and I don't want anybody to know that I've gone through those doubts." (Geraldine: TM 15759)
}

Safe environments are also those where there is space for the mentee's voice to be heard: 'It's being mindful of giving the other person, the novice, the space to explore... stuff in their own terms' (Lesley TM: 55957). Creating open communication spaces where mentees feel able to construct the meaning of issues for themselves means mentors taking a problem solving approach rather than a solutions based approach 'not just coming in and... solving everything for that person because "this is my experience, and I know that these particular things..."' (Lesley: TM55957). There is a sense of unconditionality between the mentor and mentee when they communicate in a safe environment. By virtue of beginning to walk with another, mentors and mentees will have established moderate to high levels of engagement and trust, characterised by greater disclosure by both the mentor and mentee. Fuelled by their shared values and interests their interactions can now be described as 'an 
unconditional... learning relationship that was absolutely two way' (Bella: TM 42111).

Mentoring relationships, as do all relationships, operate with a set of boundaries that are determined by the mentor initially. As time goes by, though, power relations between the two shift from being mentor driven, to reflect a balance between partner interactions, redefining the boundaries of the relationship and often the name that they use to define it.

I think... the turning point of our relationship, could have been she'd had... a shocking shift and she just felt like she had nobody else to ring but me. So I think... even though that was a mentor thing... we slipped into a friendship mode probably... because you know she was so distressed about this particular shift. (J) So that was a real demonstration of trust? (Margaret)Yeah very much so because she could have rung Mum at home. (Margaret: TM 26670)

At the point where the mentor and mentee have negotiated a safe environment for them both, the agenda of the mentoring relationship is directed by the mentee in order to meet their needs, reflecting this shift in power and the changing relations between the two: 'I'm guided by her, I let her make all the moves' (Elizabeth: TM 34898).

Conversely, a toxic, or unsafe relationship for either one or other in the mentoring partnership can occur. Toxic relationships are characterised by low levels of engagement between experienced and novice or new rural nurses during the process of getting to know a stranger. As a result levels of trust do not build and the relationship does not progress to walking with another. 'Toxic relationships do occur [but]...never get any further because they are toxic' (Bella: TM 5868).

Neither party is committed to the toxic relationship. Symptomatic of this is a reluctance to make time for communication, which is directly related to developing engagement and trust. Investing time in a mentoring relationship is a key ingredient in creating a safe environment to enable keeping things in perspective. 'I could give 
her the time. That's one of key things... if you make a commitment you've got to give them the time' (Geraldine: TM 22246).

Role modelling is another way that mentors work to keep things in perspective for mentees. This occurs under the conditions of planned face-to-face and accidental face-to-face. Mary describes role modelling as

\footnotetext{
...the more practical side of actually not do as I say, but do as I do... Because you can teach somebody something by showing them what to do and then getting them to do it and watching them do it, whereas if you try and explain to them you know you may not get the message across. (Mary: TM 22268)
}

Experienced rural nurses who are chosen as mentors by mentees are generalist, advanced practitioners who demonstrate best practice in clinical care and how they work with others in the multi-disciplinary health care team.

\footnotetext{
people who have... behaviours/characteristics or skills you want to aspire to... you think, yes that's the way I'd [like to] be able to do that, or that's the way in that situation... I'd like to be able to respond .... and it's not... necessarily someone who's your best friend, or somebody in your circle of friends within nursing. It can be somebody who people respect. (Lesley TM: 47748)
}

Rural nurse mentors term this inspirational practice "professional" behaviour and expect that through demonstrating this they will be able to keep in perspective scenarios that the mentee may initially find either confronting or challenging. 'I think the mentee is also looking for somebody that they can see professionally on another level' (Geraldine TM: 33906). Under face-to-face conditions role modelling is a tactile, visual experience as well as a verbal experience. Mentees are able to watch, listen and learn through questioning and discussion.

In all three contexts, role modelling overlaps with the construction of the mentor being a critical friend to the mentee.

Yes they need somebody... because... if they go home and say this to Mum and Dad, Mum and Dad are going to get a bit anxious that they are going to quit. Whereas somebody that is in 
the profession I think allows them to just say "let me talk about it and it's okay you can tell me what you think." Like I always relate stories how we all go through it. How we all have days and those days will come back and it is really quite normal how they're feeling and it is good that they talk about it and just re-evaluate and reassess where they are at. (Geraldine TM:

16800)

Being a critical friend, in the phenomenon of cultivating and growing rural nurses, means listening to the mentee's stories and offering positive comments and alternatives for them to consider should the situation that they talk about arise again. In the context of planned, distant relationships the construction of critical friend more aptly describes the interaction that occurs, as opposed to role modelling. Bella describes her role as a mentor in the context of planned distant as,

... the person that she could send emails to, any time she needed to, and ask any question at all... It was, (pause)... a relationship that... was not in any other part of her life... It wasn't like going and talking to... a lecturer at the uni. It was... a relationship... [where] she could ask me anything... [as] a more experienced registered nurse, she could say... "what do you think about this? or, "what happened when you did that?" and... then we would share stories. (Bella :TM 6259)

\section{Nurse Chat}

It is in the commonality of partners' perspectives of self that the foundations of the mentoring relationship are strengthened, and levels of engagement and trust increase. An outcome of recognising similarities and the bonding that occurs because of these is in the use of nurse chat in mentoring relationships. Rural nurse mentors use nurse chat when they role model and act as a critical friend in order to keep things in perspective. There is a sense of connection through the use of a common language that transcends procedural explanation, rather moving towards questioning the actuality of what it means to be a nurse. 'I think... what I meant by... nurse chat is that you... can go straight to a level of understanding because we're all nurses, we're 
a group and we don't need an explanatory note to start with before we go into a conversation' (Elizabeth: TM 17147).

Language use in cultivating and growing rural nurses is dimensionalised from superficial nurse chat to complex nurse chat. Progression along this dimension is tied to a progression along the dimension of engagement and trust. Nurse chat means that, when you actually have those conversations like that you're actually, you're actually having a conversation at a deeper personal level because in some respects you are talking about your beliefs and your values and... your feelings as well so you might not have that, well you wouldn't, have that conversation with everyone.... So you've developed a rapport with someone so now you are happy to have the conversation where it actually exposes you a bit more as a person and a practitioner.' (Elizabeth: TM 45554)

As mentors and mentees begin to engage, their language changes to reflect their joint immersion in the business of rural nursing. In the early stages of getting to know a stranger this will probably be superficial nurse chat where living out an interest in rural nursing leads to an increased familiarity with the language that rural nurses use to talk about their practice.

\footnotetext{
We could be sitting and having a cup of tea and ... a question will come up and we'll go into this... spiel about... stuff that we probably should, as nurses be talking about... and we just disappear... (J) So you have the language? (Margaret)...yeah very much. (J)... has that developed over time or do you think that that was always there? (Margaret) I think it's definitely developed over time but I think some of it was there. (Margaret: TM 33391)
}

For the novice, the mentor may also be a translator of superficial nurse chat helping them to make sense of their new practice world: ' ... she rang up and she said what does it mean when they say like a medication is... BD [twice per day] and then they have another dose that's PRN [as required]?'(Elizabeth: TM 34597). Nurse chat provides a common language that rural nurse mentors and mentees use to communicate about their practice lives on a variety of levels. 


\section{An Outcome Not An End}

Outcomes of cultivating and growing rural nurses through getting to know a stranger but particularly for walking with another are the gathering of wisdom for both mentors and mentees.

I think a mentor, you... [are] gathering wisdom [through mentoring]. It's not something that's ever going to come to an end, or it shouldn't because that's part of who you are and what you're sharing and your personality and all your history. (Bella: TM 25715)

Rural nurse mentors learn new information and gain fresh ideas from interacting with their mentees, creating win-wins situations: ' ...for me I think it's only been a win-win, its only been beneficial for me' (Bella: TM 20930). This is achieved through fostering relationships that are two-way streets: '...it's a two-way, two-way interaction. You might ... give them knowledge... but they'll give you a way of spreading it. So yeah it's a two-way street' (Kay TM: 40785).

As well, mentoring enables rural nurses to hold up a mirror to practice as they reflect upon their lives as rural nurses. Sometimes these reflections are shared through the telling of stories to their mentee; however, sometimes they just provide rural nurses with the ability to really see their practice from another perspective. Lesley likens this feeling to the story of the emperor's new clothes when the "truth" is spoken by a child, too naive to account for politics or tradition.

It is very much holding up a mirror to your own practice. And... that's really valuable. ...

And also from [my mentee's] ideas. As a novice she...comes in from left field and sometimes you think...that's such a good idea... The emperor's new clothes. (Lesley: TM 31408)

Engaging in reflection is integral to the mentoring process; those who mentor give themselves an opportunity to change or affirm their practice in light of their own selfrevelations. Rural nurse mentors also gather wisdom through watching the growth of their mentees and describe the experience as one that gives them enjoyment, and 
makes them feel honoured. 'I've actually made a difference in her life, which would make a difference in somebody else's... down the track' (Margaret: TM 42202).

Rural nurse mentors and their mentees generate and share wisdom through their interactions and it is the experience of doing so that leads them to form other mentoring relationships. Rural nurses who mentor reflect upon their experiences of being mentored and use the wisdom that was generated then, to inform the way that they mentor others and in doing so perpetrate a cycle of cultivating and growing others that is part of rural nursing practice. Such a cycle is context dependent-with varying levels of engagement and trust that will influence how it is named.

Cultivating and growing new and novice nurses inspires experienced rural nurses to be proactive in their workplace and encourages a learning environment, whereby these leaders are more open to facilitating more supportive relationships with their colleagues. The attitude that leads rural nurses to mentor novice nurses is not something that changes when a mentoring relationship finishes. Mentoring is a part of the perspective of self that they call nurse. Integrating mentoring into clinical practice is driven by how rural nurse mentors live their work as members of a rural community. For these rural nurses individual mentoring relationships provide an outcome - not an end.

\section{DISCUSSION}

\section{The Triumvirate of Nurse Mentoring: Motivation, process and outcome}

Literature about nurse mentoring abounds (Block \& Korow 2005, Carroll 2004). Results from this study affirmed Stewart and Kruger's definition of nurse mentoring with an amendment to include clinicians as potential mentees. 
within a one-to-one, reciprocal, career development relationship between two individuals diverse in age, personality, life cycle, professional status, and/or credentials. The nurse dyad relies on the relationship in large measure for a period of several years for professional outcomes, such as research and scholarship; an expanded knowledge and practice base; affirmative action; and/or career progression. Mentoring nurses tend to repeat the process with other nurses for the socialization of [clinicians], scholars and scientists into the professional community and for the proliferation of a body of nursing knowledge. (1996, p. 315)

The majority of articles written have three main elements: motivation for mentoring (Gibson \& Heartfield 2005, Heartfield \& Gibson 2005), the process of mentoring (Barker 2006) and the outcomes of mentoring (Firtko et al. 2005). Only recently has there been a shift in focus to examine the lived experience of either mentors or mentees in relation to the traditional triumvirate of nurse mentoring (Beecroft et al. 2006, Carroll 2004, Gibb et al. 2004, Gibb et al. 2006, Stewart 2006, Waters et al. 2003). These recent studies add depth to the literature as compared to the didactic nature of many of the previous publications. Findings from this study also identified elements of the triumvirate of nurse mentoring as important; however, they add a new dimension to our understanding about the lived experience of both mentors and mentees, in particular through the explication of the concept, walking with another.

\section{Keeping Things in Perspective Through Nurse Chat}

Evaluating mentees' perceptions of mentoring, Beecroft et al. found that mentors 'offer a sounding board, assist in interpreting communication, provide a different perspective and are someone to go to in times of doubt and stress' (2006, p.737). These findings are congruent with two of the subcategories of walking with another: keeping things in perspective through role modelling and the translative aspect of nurse chat.

In every study, there are always findings that hold tantalising promise for further research. Nurse chat between mentors and mentees has not previously been identified 
in studies of nurse mentoring and further research on this topic would be useful in better understanding the significance of language in developing supportive relationships.

Nursing identifies as an oral culture that relies on talk to make ordinary the often life shattering events that go on during the course of nursing work (Parker \& Gardner 1992). Nurses use of language in clinical practice has mainly been explored in relation to the process of handover (Payne \& Hardey 2000). Parker et al. maintain that 'the collaborative narrative of the handover functions to weld the nurses in to a working social group' (1992, p.32) which is important in managing the emotional impact of their work.

Our construction of nurse chat confirms the power of collaborative narrative in signifying the accepted norms and symbols of a reference group such as rural nursing. Experienced rural nurse mentors use nurse chat with new or novice rural nurse mentees to assist them to manage and better understand their working lives as members of a particular reference group.

Chatting has been identified as an important vehicle through which rapport can be established and power differentials minimised between nurses and clients (Fenwick et al. 2000). The data presented in this study adds to these findings by postulating that nursing has its own symbolic language that mentors teach their mentees through chatting together. Another important function of nurse chat is translating the meaning of language used in the clinical setting.

Nurse chat progresses from superficial to complex depending on the level of trust and engagement between the rural nurse mentor and mentee. Fenwick et al.'s argument that effective chatting establishes rapport supports this. Complex nurse chat for rural nurse mentors equates with effective nurse chat, establishing a 'two-way 
dialogue that provided the opportunity for both participants to question each other openly and share equally their life experiences' (Fenwick et al. 2000, p. 591).

\section{An Outcome Not an End}

Our construction of mentoring being an outcome not an end for the mentor adds to the common perception of mentoring being a mutual process that is beneficial for both parties (Grindel 2004, Vance \& Olson 1998). Carroll reverses this construction stating that 'nursing depends on the budding novice travelling a path which will continue to co-create a future for the discipline' (2004, p.321). This study in concert with Carroll (2004) reveals that mentoring needs to be a part of nursing practice, rather than an intervention to be applied with the aim of improving nurse retention.

Experienced rural nurses who view mentoring as an outcome not an end invest time in their relationships in order to develop sufficient levels of trust and engagement between themselves and their mentee. This is supported by an emerging argument in the literature that time is essential for successful mentoring relationships (Beecroft $e t$ al. 2006, Waters et al. 2003). The amount of time invested influences the choice of name for rural nurses' supportive relationships: preceptoring, accidental mentoring, mentoring or possibly deep friendship. We found Beecroft et al.'s study particularly interesting because it examined the mentees' experience as opposed to our study which examined mentors' experiences - and yet both sets of findings demonstrate considerable similarities.

\section{Key Points}

1. Rural nurses develop strategies to manage living and working in the same community.

2. These strategies are passed on to new or novice rural nurses in the process of walking with another. 
3. Mentoring is the name used by experienced rural nurses to name the process of walking with another.

4. Creating supportive working environments that foster experienced rural nurses mentoring new or novice nurses can impact on staff retention.

\section{Study Limitations}

This study was designed to be exploratory, descriptive and generate a theorisation about Australian rural nurses' experiences of mentoring. A possible limitation of the study originated from the initial design when we advertised for rural nurses to talk about their experiences of mentoring. Participants who volunteered had all attended development workshops facilitated by one of the researchers that had led them to define some of the supportive relationships they developed in practice as mentoring relationships. We attracted one participant who had not had any formal mentoring training through snowball recruitment; however, the participant group were on the whole well informed about the possibilities of mentoring which influenced both how they constructed their eligibility to participate and our co-constructions about their experiences.

\section{CONCLUSION}

Cultivating and growing rural nurses is a process born out of rural nurses' experiences of living their work. Working with new or novice rural nurses, they initially get to know a stranger and levels of trust and engagement between the two grow over time. Once a foundation for the relationship is established, they walk with another in order to keep things in perspective for the new or novice nurse. Sharing a language called nurse chat, the experienced rural nurse mentor takes on the role of significant other in shaping the mentee's frame of reference regarding rural nursing 
culture, politics and clinical practice. They do this in the knowledge that cultivating and growing is an ongoing process integral to their practice as rural nurse leaders.

This study is of significance to nurses generally as we struggle to provide supportive working environments, both rural and urban. Identifying experienced nurses who mentor another, as a part of their practice - affirming them and supporting them, will maintain this cycle. Mentoring new or novice nurses early in their career shapes how they frame their nursing perspective of self to aspire to be significant others over time, creating a future culture of support. More longitudinal research that gathers evidence about the impact of mentoring on nurse retention would be useful to strategically plan for the development of mentoring as a workforce support strategy that is a mainstay of contemporary nursing practice. 


\section{REFERENCES}

Barker, E. (2006) Mentoring - A complex relationship. Journal of the American Academy of Nurse Practitioners 18, 56-61.

Beecrot, P., Santner, S., Lee Lacy, M., Kunzman, L. \& Dorey, F. (2006) New

Graduate Nurses' Perceptions of Mentoring: six-year programme evaluation. Journal of Advanced Nursing 55, 736-747.

Benford, R. \& Snow, D. (2000) Framing Processes and Social Movements: An

Overview and Assessment. Annual Review of Sociology 26, 611-39.

Block, L. \& Korow, M. (2005) The Value of Mentorship Within Nursing

Communications. Nursing Forum 40, 134-140.

Blue, I. (2002) Australian Rural Health Care Professional Practice. In: Wilkinson, D. \& Blue, I. (eds.) The New Rural Health. Melbourne: Oxford.

Bushy, A. \& Leipert, B. (2005) Factors that influence students in choosing rural nursing practice: a pilot study. Rural and Remote Health 5, Online.

Carroll, K. (2004) Mentoring: A Human Becoming Perspective. Nursing Science Quarterly 17, 318-322.

Charmaz, K. (2006) Constructing Grounded Theory: A Practical Guide Through Qualitative Analysis, Thousand Oaks: Sage.

Clarke, A. (2005) Situational Analysis: Grounded Theory After the Postmodern Turn, Thousand Oaks: Sage Publications.

Corbin, J. \& Strauss, A. (1990) Grounded Theory Research: Procedures, Canons, and Evaluative Criteria. Qualitative Sociology 13, 3-21.

Dakin, S., Bryant, R. \& Foley, E. (2004) Nursing Workforce Issues in Australia. Nursing and Health Policy Review, 3, 129-140. 
Fenwick, J., Barclay, L. \& Schmied, V. (2000) 'Chatting': an important clinical tool in facilitating mother in neonatal nurseries. Journal of Advanced Nursing 33, 583-593. Firtko, A., Stewart, R. \& Knox, N. (2005) Understanding mentoring and preceptorship: Clarifying the quagmire. Contemporary Nurse 19, 32-40. Gibb, H., Anderson, J. \& Forsyth, K. (2004) Developing support for remote nursing education through workplace culture that values learning. Australian Journal of Rural Health 12, 2001-205.

Gibb, H., Forsyth, K. \& Anderson, J. (2006) Culture of Rural Nursing Practice: A Critical Theoretical Analysis of Determinants of Power in Nursing. Australian Journal of Advanced Nursing 23, 34-39.

Gibson, T. \& Heartfield, M. (2005) Mentoring for nurses in general practice: an Australian study. Journal of Interprofessional Care 19, 50-62.

Glaser, B. \& Strauss, A. (1967) The Discovery of Grounded Theory, New York: Aldine De Gruyter.

Glass, N. \& Walter, R. (2000) An experience of peer mentoring with student nurses: enhancement of personal and professional growth. Journal of Nursing Education 39, $155-60$.

Grindel, C. (2004) Mentorship: A Key to Recruitment and Retention. Medsurg Nursing 13, 36-37.

Hanson, C. M. \& Hilde, E. (1989) Faculty mentorship: support for nurse practitioner students and staff within the rural community health setting. Journal of Community Health Nursing 6, 73-81.

Heartfield, M. \& Gibson, T. (2005) Mentoring for nurses in general practice: national issues and challenges. Collegian 12, 17-21. 
Hegney, D., McCarthy, A., Rogers-Clark, C. \& Gorman, D. (2002) Why nurses are resigning from rural and remote Queensland health facilities. Collegian: Journal of the Royal College of Nursing, Australia 9, 33-9.

Koch, T. (2006) Establishing rigour in qualitative research: the decision trail. Journal of Advanced Nursing 53, 91-103.

Lindsay, D., Malko-Nyan, K., Gilmore, V., Van Haaran, M., Watts, J., Knight, S., Francis, K., Fletcher, J., Goold, S., Brans, L., Siegloff, L., Veitch, C. \& Anm, R. (2002) Action on Nursing in Rural and Remote Areas Recommendations and Action Plan. Canberra: National Rural Health Alliance.

Mills, J., Bonner, A. \& Francis, K. (2006) Adopting a Constructivist Approach to Grounded Theory: Implications for Research Design. International Journal of Nursing Practice 12, 8-13.

Mills, J., Francis, K. \& Bonner, A. (2006) The problem of workforce for the social world of Australian rural nurses: a collective action frame analysis. Journal of Nursing Management, 14, 1-11.

Mills, J., Francis, K. \& Bonner, A. (2005a) Mentoring, clinical supervision and preceptoring: clarifying the conceptual definitions for Australian Rural Nurses. A review of the literature. Rural and Remote Health 5, Online.

Mills, J., Lennon, D. \& Francis, K. (2005b) Mentoring Matters: developing rural nurses knowledge and skills. Collegian 13.

Parker, J. \& Gardner, G. (1992) The silent and the silencing of the nurse's voice: a reading of patient progress notes. The Australian Journal of Advanced Nursing 9, 3-9. Parker, J., Gardner, G. \& Wiltshire, J. (1992) Handover: the collective narrative of nursing practice. The Australian Journal of Advanced Nursing 9, 31-37. Payner, S. \& Hardey, M. (2000) Interactions between nurses during handovers in elderly care. Journal of Advanced Nursing 32, 277-285. 
Stewart, B. M. \& Krueger, L. E. (1996) An evolutionary concept analysis of mentoring in nursing. Journal of Professional Nursing 12, 311-21.

Stewart, D. (2006) Generational Mentoring. The Journal of Continuing Education in Nursing, 37, 113-120.

Strauss, A. \& Corbin, J. (1990) Basics of Qualitative Research: Grounded Theory Procedures and Techniques, Newbury Park: Sage Publications.

Strauss, A. \& Corbin, J. (1998) Basics of Qualitative Research: Techniques and Procedures for Developing Grounded Theory, Thousand Oaks: Sage Publications. Vance, C. \& Olson, R. (1998) The Mentor Connection in Nursing, New York: Springer Publishing.

Waters, D., Clarke, M., Harris Ingall, A. \& Dean-Jones, M. (2003) Evaluation of a pilot mentor programme for nurse managers. Journal of Advanced Nursing 42, 516526. 